\title{
Defining Language Learning Strategies: Implications for Research
}

\author{
Zahra EL AOURI, \\ Doctorate in Education, Mohammed V University - Souissi Faculty of Education - Rabat - Morocco
}

\begin{abstract}
This paper addresses some controversial issues in defining language learning strategies (LLSs). Three major points will be discussed. First, some thorny issues related to the definition of language learning strategies will be raised. The focus will be on the issue of the definitions of LLSs; the terminology/taxonomy used to refer to these strategies; the criteria used to classify them; and the conscious/unconscious nature of LLSs. Second, there will be an open-question as to whether there should be a clear-cut definition of LLSs in the literature. The third and last point of focus in this paper will discuss the impact of the problematic definitions of LLSs on the choice of data collection procedures.
\end{abstract}

Keywords: LLSs, language learning strategies, strategy terminology, strategy taxonomy, conscious/unconscious strategies

\section{Introduction}

Preliminary research on LLSs began in the early 1970's with Rubin (1975); then later this field of research became prolific, especially with the emergence of the notion of "the good language learner" (Rubin, 1975; Naiman, Frohlich, Stern and Todesco, 1978). In the 1980's and 1990's LLSs knew a boost in research studies which dealt with LLSs from different perspectives and theoretical frameworks.

However, this field still suffers from different debatable issues especially with respect to the plethora of definitions provided by a number of researchers. These different definitions make it problematic to provide both a clear terminology and taxonomy of LLSs. All this has led to the rise of the conscious/unconscious dichotomy of LLSs and the differing criteria used to classify them. The discussion of these debatable issues related to the study of LLSs will lead us to reflect on whether it is possible to come up with an agreed-upon definition of LLSs that can bring together the various definitions provided in the literature. Not only do the various definitions of LLSs have an impact on the terminology and taxonomy used to refer to them, but they affect the choice of data collection procedures selected to study LLSs as well.

\section{Thorny issues in defining LLSs}

Although many studies have contributed to the richness of language learning strategies, there are still persistent debatable issues pertinent to definitions and terminology/taxonomy.

\subsection{Definitions of LLSS}

The literature on LLSs reflects the extent to which the definition of these strategies is so ambiguous and "loose" as it is bunched with all types of learner behaviors. This ambiguous nature, lack of clarity and the broad definition of what a strategy really is makes of strategies a "fuzzy" concept. In fact, strategies are most of the time defined so broadly that it is difficult to classify them as observable, non-observable, specific or universal behaviors.

The following are the major definitions provided by experts in the domain:

Rubin refers to LLSs as "any set of operations, plans, or routines, used by learners to facilitate the obtaining, retrieval, storage and use of information" (1987, p.19)

Rigney (1978) defines in broad terms LLSs as "a set of operations used by the learner to aid the acquisition, storage and retrieval of information".

However, Stern states in somewhat specific terms that "the concept of learning strategy is dependent on the assumption that learners consciously engage in activities to achieve certain goals and learning strategies can be regarded as broadly conceived intentional directions and learning techniques." (1992, p.261)

Cohen shares some ground with Stern when he says that "strategies can be classified as conscious mental activity. They must contain not only an action but a goal (or an intention) and a learning situation.

Whereas a mental action might be subconscious, an action with a goal/intention and related to a learning situation can only be conscious." (2007, p.31) 
O'Malley meets with Cohen in choosing the terms "conscious" and "goal" in defining LLSs when she refers to LLSs as "The conscious thoughts and actions that learners take in order to achieve a learning goal" (2004, p. 14).

Oxford developed a more comprehensive definition of LLSs as she claims that they are "...operations employed by the learner to aid the acquisition, storage, retrieval and use of information; specific actions taken by the learner to make learning easier, faster, more enjoyable, more self-directed, more efficient, and more transferable to new situations." (2001, p. 166).

From this limited set of definitions one can clearly notice the inconsistency between researchers with regard to LLSs definitions. Some researchers refer to strategies as operations and plans which imply the concept of strategy while others refer to strategies as actions and activities which imply the notion of process more than only strategy. Nevertheless, some researchers seem to agree directly or indirectly on the conscious nature of LLSs along with some goal-oriented learning situation involved in the use of LLSs. Still researchers put emphasis on the cognitive side of LLSs and the information processing activities carried out by learners when using LLSs. They almost all refer to strategies as a facilitator of acquisition, storage/retrieval and use of information.

\section{Terminology/taxonomies used to refer to LLSs}

In the same way definitions of LLSs are so varied and overlapping according to the different theoretical frameworks from which researchers treat LLSs so is the terminology used to refer to these strategies and the taxonomies used to classify them.

\subsection{Terminology of LLSs}

The theoretical frameworks underlying LLSs range from educational psychology, cognitive psychology and cross-cultural psychology to information-processing theory to name but a few; and these frameworks explain why researchers espouse different terminologies in their research studies on LLSs. Therefore, learning strategies are a fuzzy concept and, hence, used interchangeably with the following terms: learning behaviors, cognitive processes and tactics as Larsen-Freeman and Long (1991) claim. Wenden $(1987,1991)$ also notes that other terms are used synonymously to refer to learning strategies such as techniques, potentially conscious plans, consciously employed operations, learning skills, cognitive abilities, processing skills, problem-solving procedures and basic skills. For Oxford (1990b), learning strategies are also equated with thinking skills, thinking frames, reasoning skills, tactics and learning to learn skills.

\subsection{Taxonomies of LLSS}

There are several taxonomies to classify LLSs, but the focus here will be on four taxonomies or classifications:

1. O'Malley's (1985) taxonomy: O'Malley et. al. (1985, p.582-584) categorize LLSs into three main categories:

1.1 Metacognitive strategies which require planning for learning, thinking about the learning process as it is taking place, monitoring one's production or comprehension; and evaluating learning.

1.2 Cognitive strategies which are more limited to specific tasks and involve a degree of manipulation of the learning material.

1.3 Socio-affective strategies which are related to social-mediating activities and cooperating with others.

2. Rubin's (1987) taxonomy: Rubin distinguishes between strategies which directly contribute to language learning and those which indirectly contribute to learning. He lists three types of strategies:

Learning strategies which include two types: cognitive learning strategies and metacognitive learning strategies. Both of these strategies contribute directly to the development of the language system constructed by the learner.

Communicative strategies which relate less to language learning because their focus is on the process of participating in communication and getting the meaning across without losing the communication exchange due to some lack of language knowledge.

Social strategies which relate indirectly to language learning but help learners engage in activities and provide them with opportunities to practice their knowledge of the target language.

3. Stern's (1992) taxonomy: Stern postulates five main categories (p.262-266):

Management and planning strategies which relate to setting goals for learning; selecting appropriate resources; committing oneself to learning; and evaluating achievement.

Cognitive strategies which are operations used in learning or problem-solving situations.

Communicative-experiential strategies which consist of strategies to help learners communicate with the degenerate input they have and socially interact with others. 
Interpersonal strategies which help learners contact native speakers and become acquainted with the target language culture.

Affective strategies which are used to regulate and monitor emotional difficulties while learning a language.

4. Oxford's (2001) taxonomy: for Oxford, the aim of LLSs is the orientation towards the development of communicative competence. She classifies strategies into two main categories: direct and indirect strategies which are in turn subdivided into six subcategories (Oxford, 2001, p.359):

Cognitive strategies which enable the learner to manipulate the target language material in direct ways such as note-taking, reasoning, analyzing, summarizing, synthesizing, outlining ...etc

Metacognitive strategies which enable the learner to manage the learning process through identifying the learning style preferences, planning for L2 learning, gathering and organizing materials, arranging for learning, monitoring mistakes, evaluating the learning process ... etc

Memory strategies which enable the learner to link one L2 item or concept with another.

Compensatory strategies which help the learner make up for missing knowledge such as guessing from context in listening and reading, using gestures ... etc

Affective strategies which enable learners to control their mood and anxiety level, talk about feelings, reward oneself for good performance, use positive self-talk and deep breathing ... etc

Social strategies which help the learner work with others and cooperate to understand the target language as well as the culture. For instance, learners ask questions, seek verification, ask for clarification ... etc

The most exclusive taxonomy is the one developed by Oxford because it reflects validity for research and brings the other taxonomies together. That is, Oxford's taxonomy reflects the intersection of the different taxonomies already mentioned.

\section{Criteria used to classify LLSs}

There is a crucial problem in the field of LLSs which is related to the use of different criteria to classify them. This causes mismatches and inconsistencies across existing taxonomies of LLSs. The first criterion is the direct/indirect dichotomy used to classify strategies; some strategies have a direct impact on improving learning while others contribute indirectly to language learning. The second criterion is related to observable/nonobservable behaviors in a strategy; some strategies are behavioral and, hence, can be directly and easily observed whereas others are mental strategies and cannot be easily observed. The third criterion is that strategies are classified according to the dichotomy successful/unsuccessful language learners. This is quite debatable since the effectiveness of a given strategy depends largely on the characteristics of the learners, the language structure, the context or the interaction of all these. The last criterion to classify strategies is that close terms are used to categorize them as cognitive, metacognitive, affective or social. The problem is that the distinctions are not all the time clear-cut because sometimes they overlap.

\section{Conscious/unconscious nature of LLSs}

In the literature, there are groups of researchers who state that consciousness is an essential feature of a strategy; while other groups just disagree with this statement. Since strategies have a goal, which is to facilitate learning, so they must be conscious and intentional. According to Cohen (1990) strategies are learning behaviors which are consciously selected by the learner. Cohen claims that conscious choice is important as it is a special feature of strategy. Moreover, Cohen refers to strategies as "moves which the learner is at least partially aware of, even if full attention is not being given to them. There must be some level of awareness that a strategy is being used. If a learner's behavior is totally unconscious, then it would simply be referred to as a process, not a strategy." (1990, p.11). Here Cohen calls upon the dichotomy of strong and peripheral attention.

However, there are other researchers who assert that strategies do not involve consciousness or awareness on the part of the learner. For example, Bialystok (1990) admits that all definitions of strategies involve consciousness and intentionality; and at the same time, however, she contradicts these definitions by saying that strategies cannot have consciousness as a criterion.

Although O'Malley and Chamot (1990) strongly support Weinstein and Meyer's (1986) statement that learning strategies being intentional and conscious, they underscore the strong possibility that LLSs can be unconsciously performed because what is conscious and intentional becomes an automatic process.

Therefore, there is no clear distinction between conscious and unconscious nature of LLSs but the use of the latter becomes so natural and automatic to the extent that it becomes unconscious. To deal with the dichotomy of conscious/unconscious nature of LLSs, researchers resorted to language learning strategy training which asserts that LLSs are indeed conscious behaviors or actions taken to improve language learning. This implies that if LLSs were unconscious actions, strategy training would be useless. 


\section{An open question on an agreed-upon definition of LLSs}

Should there be a clear-cut and agreed-upon definition of language learning trategies? This is the question that any one interested in LLSs should ask.

While surveying the literature on LLSs, one can notice that there is no one and clear definition shared between researchers. This is quite understandable if we take into consideration the fact that researchers provide definitions of LLSs with respect to the theoretical framework from which they deal with learning strategies.

However, researchers tried to focus on Oxford's definition because they found it comprehensive and it shares many features with the other definitions provided by different researchers. That is, her definition highlights the conscious side of LLSs; the cognitive and metacognitive nature of those strategies; the mental operations involved in LLSs; the goal-oriented characteristic; the facilitating role of strategies; the ability of LLSs to develop autonomy in learners who use them; and the transferability of those strategies to new learning situations.

Having synthesized the major definitions of LLSs in the first part of this paper, now I will try to select the terms that intersect in the different definitions and come up with a definition of LLSs.

"Language learning strategies are plans, mental operations and conscious, goal-oriented actions that learners engage in while learning a language. These actions can facilitate the acquisition, storage, retrieval, and use of information by the learner. They are even transferable to new situations of language learning. Moreover, if learners really perform these actions regularly, they will become more autonomous, more self-directed and involved in language learning."

The choice of this definition stems from the fact that it encompasses nearly all the definitions provided by the different researchers in LLSs cited earlier in this paper.

\section{The impact of the problematic definitions of LLSs on the choice of data collection procedures}

Defining LLSs requires a clear vision of the latter. However, the problem that researchers face is first identifying a strategy; second describing this strategy; and finally giving it a definition. Since there is no clearcut and shared definition of LLSs between researchers, the choice of instruments to measure LLSs and collect data remains open to question. Differently put, the fuzzy nature of LLSs has a great impact on the selection of instruments for data collection.

In the field of LLSs different methods have been used to collect data depending on the objectives of the research study. The focus in this section will be on verbal report protocols, questionnaires, interviews and observation, beside other methods.

According to Cohen verbal report protocols include data that reflect self-report, self-observation (introspective/retrospective), and self-revelation (think-aloud or stream-of-consciousness methods) (1996, p.13) With respect to LLSs, verbal report protocols are extensively used in researching strategies depending on the language skill being investigated. In fact, they are used especially in written communication skills (reading and writing). However, there are some problems with verbal reports. For example, cognitive processes may not be fully conscious and, hence, learners may miss to verbalize their thoughts. Moreover, verbal reports may disrupt normal behavior in that learners have to stop each time and talk about what is going on in their minds. Besides, learners may not know how to do verbal reports and, therefore, need more practice which may have an effect on the intended data. Despite all these problems, verbal report protocols remain effective in helping learners provide the data directly since the information comes during or right after the task has been performed.

Besides verbal report protocols, there is a number of questionnaires used in the literature of LLSs. These questionnaires focus on some language skills and different strategies. However, most of them suffer from problems of research validity and reliability. For this reason, the need for a more comprehensive scale to measure LLSs emerged. This scale is the SILL - the Strategy Inventory for Language Learning - which was developed by Oxford (1986-1990). This is the only standardized questionnaire that has been extensively checked for reliability (.94 Cronbach Alpha reliability index; Oxford, 1996, p.32) and validated in multiple ways as it has been and still is used across the world in many studies. Furthermore, the SILL is a non-threatening instrument since it is usually administered under conditions of confidentiality. It is also free of social desirability response bias (Oxford, 1996).

Interviews are another instrument for data collection on LLSs. They can be either structured or semistructured. Interviews are used to identify strategies employed in specific tasks over a period of time, but they are less useful for identifying typical strategies because it all depends on how interviews are conducted and how questions are asked.

Another instrument that has been used to study strategies is observations which the researcher carries out while learners are engaged in a task in the classroom. Observations can range from either formal or structured (through observation grids) to informal classroom observations. The problem with observations, however, is that some strategies are internal, mental in nature and, hence, unobservable. Moreover, observations can rely on the more outspoken, extrovert learners in the classroom without focusing on the introvert ones. Still 
observations can provide data on observable strategies to complement the other methods such as verbal reports and strategies can be assessed on the spot as tasks are performed (Oxford, 1996) There are of course other instruments to collect data on LLSs such as diaries, recollective narratives, strategy checklists and computer tracking.

\section{Conclusion}

Research on LLSs provides different definitions of LLSs depending on the theoretical framework used to investigate strategies. Research in this field suffers from some thorny issues related to definitions of LLSs; terminology/taxonomy used to refer to these strategies; the criteria used to classify strategies; and the conscious/unconscious dichotomy in LLSs. Having dealt with these debatable issues, the question arises as to whether there is a clear-cut and shared definition between researchers. The last point is the impact of the fuzzy concept of LLSs on the choice of data collection procedures.

\section{References}

[1]. Rubin, J. (1975). What the "good language learner" can teach us. TESOL Quarterly, Vol. 9 (1), pp.41-51

[2]. Naiman, N.,Frohlich, M., \&Todesco, A. (1978). The good language learner. Research in Education Series, 7. Toronto: Ontario Institute for Studies in Education

[3]. Rubin, J. (1987). Learner strategies: "Theoretical assumptions, research History, and typology". In A. Wenden \& J. Rubin (Eds.), Learner strategies in language learning (pp. 15-30) Englewood Cliffs, NJ: Prentice-Hall

[4]. Rigney, J.W. (1978). "Learning strategies: a theoretical perspective". In: O’Neil, H.F. Jr. (Ed.) Learning Strategies (pp. 165 - 205). Academic Press, New York

[5]. Stern, H. H. (1992). Issues and options in language teaching. Oxford, UK: Oxford University Press

[6]. Cohen, A. D. (2007). "Coming to terms with language learner strategies: Surveying the experts". In A. D. Cohen \& E. Macaro (Eds.), Language learner strategies: 30 years of research and practice (pp. 29-45). Oxford: Oxford University Press

[7]. O'Malley, A.U. (2004). Issues in language learning strategy research and teaching. Electronic Journal of Foreign Language Teaching 2004, vol. 1 (1), pp. 14 - 26 available from: [http://e-flt.nus.edu.sg/V1n12004/chamot.htm] Accessed $16^{\text {th }}$ May, 2010

[8]. Oxford, R. L. (2001). "Language learning styles and strategies". In M. Celece-Murcia (Ed.), Teaching English as a Second or Foreign Language ( $3^{\text {rd }}$ ed.) (pp. 359-366). Boston: Heinle \& Heinle. Thompson International

[9]. Larsen-Freeman, D. \& Long, M. (1991). Introduction to Second Language Acquisition Research. London: Longman

[10]. Wenden, A. (1987). Incorporating learner training in the classroom. In: Wenden, A. \& Rubin, J. (Eds.) Learner Strategies in Language Learning. (pp. 159 - 168). Englewood Cliffs, NJ: Prentice-Hall

[11]. Wenden, A. (1991). Learner Strategies for Learner Autonomy. Englewood Cliffs, NJ:Prentice-Hall

[12]. Oxford, R. L. (1990b). Language learning strategies: What every teacher should know. New York: Newbury House / Harper \& Row. Now Boston: Heinle \& Heinle

[13]. O’Malley, J.M.; Chamot, A.U.; Stewner-Manzanares, G.; Russo, R.P. \&Kupper, L. (1985). Learning strategy application with students of English as a second language. TESOL Quarterly, Vol. 19 (3) 557 - 584

[14]. Cohen, A.D. (1990). Language Learning: Insight for Learners, Teachers, and Researchers. New York: Newbury House/Harper \& Row. Now Boston: Heinle\&Heinle

[15]. Bialystok, E. (1990). Communicative Strategies: A Psychological Analysis of Second Language Use. Oxford: Basil Blackwell

[16]. O’Malley, J., \& Chamot, A. (1990). Learning strategies in second language acquisition. New York, NY: Cambridge University Press

[17]. Weinstein, E. C. \& Mayer, R. E. (1986). The Teaching of Learning Strategies. Handbook of Research to Teaching. (Ed. Wittrock, M. C.) (pp. 315-327). New York: MacMillan.

[18]. Cohen, A.D. (1996). Verbal reports as a source of insights into second language learner strategies. Applied Language Learning, Vol. 7 (1\&2), pp.5-24

[19]. Oxford, R.L. (1986). Development and psychometric testing of the Strategy Inventory for Language Learning (SILL). ARI Technical Report 728. Alexandria, VA; Army Research Institute. Appendices as ARI Research Note 86 - 92

[20]. Oxford, R. L. (1990). Language Learning Strategies: What Every Teacher Should Know. Newbury House, New York

[21]. Oxford, R.L. (1996). Employing a questionnaire to assess the use of language learning strategies. Applied Language Learning, Vol. $7(1 \& 2)$, pp. $25-45$ 